\title{
EMPREGO DE FERRAMENTAS NUMÉRICAS NA AVALIAÇÃO DO MÓDULO DE ELASTICIDADE EM VIGAS ROLIÇAS DE MADEIRA
}

\author{
ANDRÉ L. CHRISTOFORO ${ }^{1}$, ANDRÉ L. ZANGIÁCOMO², TÚLIO H. PANZERA ${ }^{3}$, \\ MÁRCIO E. SILVEIRA ${ }^{4}$, FRANCISCO A. R. LAHR ${ }^{5}$
}

\begin{abstract}
RESUMO: Vigas são elementos estruturais encontrados na maioria das construções civis. Dentre os materiais de engenharia, destaca-se a madeira, por ter resistência mecânica satisfatória aliada a baixa densidade. A madeira roliça apresenta-se como boa solução na confecção de vigas, uma vez que não precisa ser processada, como é o caso da madeira serrada. O projeto de elementos estruturais de madeira requer o conhecimento de suas propriedades físicas e mecânicas, obtidas segundo as premissas de documentos normativos. Em se tratando da madeira roliça, os documentos normativos nacionais que tratam da determinação das propriedades de resistência e rigidez estão vigentes há mais de vinte anos sem revisão técnica. De forma geral, tanto as normas nacionais como as internacionais idealizam geometria troncocônica para as peças roliças de madeira, implicando equações simplificadas incapazes de prever a influência das irregularidades da forma na determinação do módulo de elasticidade longitudinal. Este trabalho objetiva avaliar a influência das irregularidades da geometria em peças roliças de madeira Corymbia citriodora e Pinus caribaea no cálculo do módulo de elasticidade longitudinal. Para tanto, utilizou-se do ensaio de flexão estática a três pontos, considerando também um modelo matemático simplificado, assumindo seção circular constante para a forma do elemento. As irregularidades das peças são consideradas nos modelos numéricos, constituídos de elementos finitos de barra e tridimensionais. Os resultados encontrados revelam equivalência estatística entre os módulos de elasticidade para ambas as formas de cálculo, indicando ser plausível a consideração de seção circular constante para as peças de madeira aqui avaliadas.
\end{abstract}

PALAVRAS-CHAVE: madeira roliça, Método dos Elementos Finitos, Método dos Mínimos Quadrados.

\section{USE OF NUMERICAL TOOLS IN THE EVALUATION OF THE LONGITUDINAL MODULUS OF ELASTICITY IN ROUND TIMBER BEAMS}

\begin{abstract}
Beams are structural elements used in the most of civil constructions. The wood can be found in several applications of engineering due to the moderate mechanical strength and low density. The use of round timber as a beam is very attractive, since it does not need to be processed, such as lumber. The design of structural timber elements requires the determination of its physical and mechanical properties in which are obtained based on the recommendations of engineering standards. In case of round timber, the national standards dealing with the determination of strength and stiffness properties are in term for more than twenty years with no technical review. Overall, both national and international standards consider truncated-cone geometry for cylindrical logs of wood, resulting in simplified equations unable to predict the effect of shape irregularities on the longitudinal modulus of elasticity. This paper aims to evaluate the effect of shape irregularity of round timber of Corymbia citriodora and Pinus caribaea to determine the longitudinal modulus of elasticity. The three-point bending test is used to determine the modulus, considering a simplified analytical model, with constant circular section for the element. The irregularities of the wood are considered in the numerical models based on a beam and three-dimensional finite elements. The results showed statistical equivalence between the modulus of elasticity for both methods of calculation, indicating that the constant circular section is a reasonable assumption for the wooden here evaluated.
\end{abstract}

KEYWORDS: round timber, Finite Element Method, Least Square Method.

\footnotetext{
${ }^{1}$ Depto. de Engenharia Mecânica (DEMEC), Universidade Federal de São João del-Rei (UFSJ), alchristoforo@ yahoo.com.br.

${ }^{2}$ Depto. de Engenharia (DEG), Universidade Federal de Lavras (UFLA), andrezangiacomo@ deg.ufla.br.

${ }^{3}$ Depto. de Engenharia Mecânica (DEMEC), Universidade Federal de São João del-Rei (UFSJ), tuliopanzera@ hotmail.com.

${ }^{4}$ Depto. de Engenharia Mecânica (DEMEC), Universidade Federal de São João del-Rei (UFSJ), msilveira@ufsj.edu.br.

${ }^{5}$ Professor Titular, Departamento de Engenharia de Estruturas, Escola de Engenharia de São Carlos, USP, frocco@sc.usp.br.

Recebido pelo Conselho Editorial em: 11-1-2012

Aprovado pelo Conselho Editorial em: 12-6-2012
}

Eng. Agríc., Jaboticabal, v.32, n.5, p.971-980, set./out. 2012 


\section{INTRODUÇÃO}

Em função da versatilidade e da disponibilidade, há séculos a madeira vem sendo utilizada como material estrutural. Em países com tradição no emprego da madeira em estruturas, é comum a utilização de sistemas mistos, tanto de madeira maciça quanto de seus derivados. Entretanto, as demandas associadas aos custos do processamento motivam pesquisas por busca de soluções que aliem alta eficiência da madeira como elemento estrutural a um baixo custo de produção. Uma alternativa a este problema é a utilização desse material em sua forma roliça original, decorrente do crescimento natural da árvore, como demonstra PARTEL (1999), num estudo que consistiu em um levantamento dos principais sistemas estruturais de habitações, edificações, torres de eletrificação e pontes utilizando madeira roliça no Brasil e no exterior.

A Norma Brasileira para o Projeto de Estruturas de Madeira NBR 7190/1997 especifica os elementos roliços a partir dos diâmetros de base e de topo, independentemente da espécie utilizada. Este documento determina, ainda, que as propriedades de resistência e rigidez sejam obtidas por meio de ensaios em corpos-de-prova de pequenas dimensões e isentos de defeitos, mesmo sendo conveniente a análise da peça com dimensões estruturais.

A verificação das diferenças existentes nas propriedades mecânicas obtidas entre corpos de prova e peças de dimensões estruturais é tema para o desenvolvimento de uma grande gama de pesquisas, podendo-se citar as de BATISTA et al. (2000), MINÁ et al. (2004) e CORSINI et al. (2004).

Países com tradição no uso da madeira para os mais variados fins apresentam um leque maior de documentos normativos quando comparados a países que ainda caminham para se firmar nesse cenário. Em se tratando da determinação das propriedades mecânicas, especificações e padronizações de metodologias experimentais para madeira de dimensões estruturais, alguns documentos normativos internacionais podem ser citados, tais como a norma ASTM D198/1976, AS 2209/1994, ASTM D1036/1997, ANSI O5.1/2002 e DIN EN 14251/2004. Os módulos de elasticidade, segundo estas normas, podem ser calculados através de ensaios de flexão em três ou quatro pontos ou na condição de vigas engastadas em balanço. Cabe ressaltar que as equações que permitem calcular o módulo de elasticidade para elementos roliços consideram a hipótese de que a peça seja troncocônica, utilizando um ou dois valores de circunferência para a determinação do momento de inércia.

No Brasil, os documentos que tratam de elementos estruturais roliços são direcionados principalmente para atender ao mercado de postes, estando vigentes há pelo menos 20 anos sem revisão técnica (CHRISTOFORO, 2011).

A norma NBR 6231/1980 (Postes de Madeira: Resistência à Flexão) prescreve o modo pelo qual deve ser feito o ensaio de resistência à flexão de postes de madeira. Uma das extremidades do elemento é engastada e, na outra extremidade, é aplicada uma força, gerando um deslocamento. Através de uma equação, em que são considerados fatores como a força aplicada, características geométricas e o deslocamento medido, determina-se o módulo de elasticidade da peça.

A norma NBR 8456/1984 (Postes de Eucalipto Preservado para Redes de Distribuição de Energia Elétrica) estabelece as condições para a preparação e o recebimento de postes de Eucalipto preservados sob pressão, para serem empregados em redes aéreas de distribuição de energia elétrica.

A norma NBR 8457/1984 (Postes de Eucalipto Preservado para Redes de Distribuição de Energia Elétrica: Dimensões) padroniza os postes de Eucalipto preservado também para serem empregados em redes aéreas de distribuição de energia elétrica. São especificados: comprimento do poste, tipo, resistência nominal, flecha máxima, comprimento e diâmetro de engastamento e perímetros de topo e de base. 
A norma técnica NBR 6122/1996 (Projeto e Execução de Fundações) recomenda o uso da norma NBR 7190/1997 para a o cálculo da resistência de estacas de madeira, sendo que esta última se limita a ensaios de resistência em corpos de prova de pequenas dimensões e isentos de defeitos.

Assim como os documentos normativos internacionais, os nacionais que tratam da madeira roliça também se fundamentam na hipótese de conicidade da forma, mesmo sabendo da existência de possíveis irregularidades na geometria da peça.

Com relação ao emprego e ao estudo das propriedades de resistência e rigidez para peças roliças estruturais de madeira, alguns trabalhos podem ser citados, como os de RANTA-MAUNUS (2000), WOLF \& MOSELEY (2000), ROSS et al. (2001), PINTO NETO et al. (2004), SALES et al. (2004), CALIL JR et al. (2004), LARSON et al. (2004), MINÁ (2005), MINÁ \& DIAS (2008), ZANGIÁCOMO \& ROCCO LAHR (2008), CARREIRA \& DIAS (2009) e SALES et al. (2010).

Dessa forma, faz-se necessário o desenvolvimento de pesquisas para que sejam determinadas, de modo confiável, as propriedades de resistência e rigidez de elementos estruturais roliços como subsídios fundamentais para produtores e engenheiros.

Este trabalho objetiva apresentar uma metodologia de cálculo para a determinação do módulo de elasticidade longitudinal em vigas roliças estruturais de madeira, sendo utilizadas as espécies Corymbia citriodora e Pinus caribaea, levando-se em consideração a influência das possíveis irregularidades da geometria. Para tanto, utilizou-se do ensaio de flexão estática a três pontos, sendo avaliado, também, um modelo matemático analítico que considera seção circular constante para a peça, e dois numéricos, com o uso de elementos finitos uni e tridimensionais, que permitem delinear com maior precisão a forma dos elementos roliços, considerando a geometria com aproximação linear e não linear por partes, objetivando-se verificar a precisão da aproximação contida no modelo analítico simplificado para com as estratégias numéricas aqui utilizadas.

\section{MATERIAL E MÉTODOS}

Para a determinação do módulo de elasticidade longitudinal, foram utilizadas 24 peças roliças estruturais de madeira Corymbia citriodora, com comprimento médio de 550 centímetros e 24 peças de Pinus caribaea, com comprimento médio de $750 \mathrm{~cm}$, ambas com conicidade média de 0,01 .

As peças roliças respeitam a relação $\mathrm{L}_{\mathrm{eq}} \geq 21$ (ZANGIÁCOMO \& ROCCO LAHR, 2008), validando o emprego da teoria de vigas de Bernoulli, sendo $\mathrm{L}$ o comprimento e $\mathrm{D}_{\text {eq }} \mathrm{o}$ diâmetro equivalente, medido no ponto médio do elemento, assumindo-se que as seções são perfeitamente circulares, que os diâmetros variam linearmente no comprimento e que o deslocamento máximo do elemento ocorre no ponto de aplicação da força (pequena conicidade).

O módulo de elasticidade, neste trabalho, é avaliado segundo dois modelos numéricos de cálculo distintos, juntamente com um analítico advindo da resistência dos materiais.

No modelo analítico (Figura 1), o módulo de elasticidade é calculado com o auxílio da eq.(1), sendo $F$ a força aplicada, $E$ o módulo de elasticidade longitudinal e $\delta$ o deslocamento linear medido abaixo do ponto de aplicação da força.

$$
E=\frac{4 \cdot F \cdot L^{3}}{3 \cdot \pi \cdot \delta \cdot D_{e q}{ }^{4}}
$$




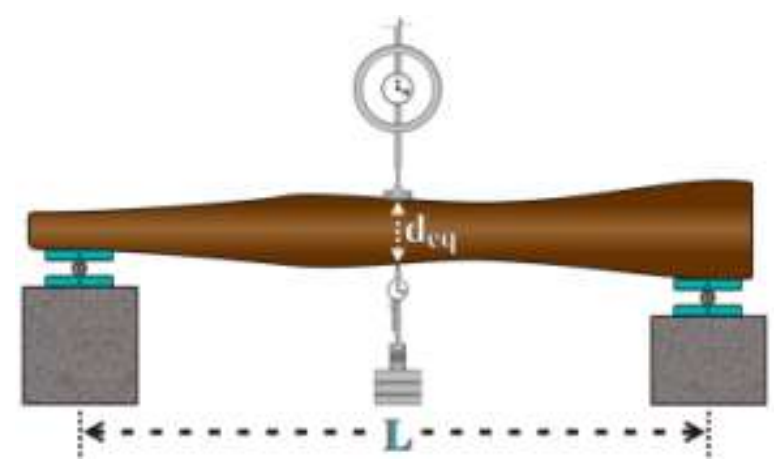

FIGURA 1. Ensaio de flexão a três pontos, segundo o diâmetro equivalente. Three points bending test according to the equivalent diameter.

Os modelos numéricos diferenciam-se pelos elementos finitos utilizados, sendo dois de vigas (elemento de barra) e um tridimensional (tetraédrico).

O esquema de ensaio utilizado no cálculo do módulo de elasticidade longitudinal, com o uso de elementos finitos de viga, é ilustrado na Figura 2.

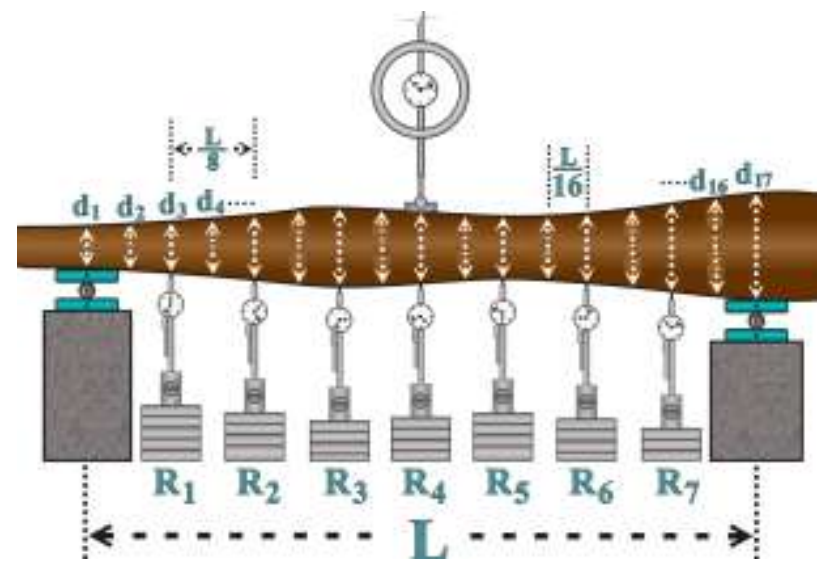

FIGURA 2. Ensaio alternativo para a determinação do módulo de elasticidade efetivo. Alternative test to determine the efective modulus of elasticity.

Na determinação do módulo de elasticidade efetivo, para cada ensaio estrutural realizado, foram locados sete relógios comparadores ao longo das peças, distantes L/8 uns dos outros, e, também, foram medidos dezessete valores de circunferência ao longo de seu comprimento, objetivando-se determinar os respectivos diâmetros $\left(\mathrm{d}_{\mathrm{i}}\right)$ e o delineamento da forma, por aproximações lineares e não lineares, sendo utilizados nove valores de diâmetros para a aproximação linear e dezessete para a aproximação não linear da geometria.

As leituras dos deslocamentos nos relógios R1, R2, R3, R5, R6 e R7 (Figura 2) são realizadas quando o deslocamento no meio do vão (R4) é da ordem de L/200, sendo L expresso em $\mathrm{cm}$. Este valor garante o comportamento elástico-linear do material e linearidade geométrica para a peça, tratando-se de uma medida para pequenos deslocamentos (NBR 7190/1997). A imposição desta relação permite que os ensaios de flexão sejam executados de forma não destrutiva (CHRISTOFORO et al., 2011).

Para o cálculo dos módulos de elasticidade efetivos, foram desenvolvidos dois programas computacionais, EotmL (aproximação linear) e EotmN (aproximação não linear), fundamentados no Método dos Elementos Finitos (MEF), segundo o emprego do modelo cinemático de deformação de vigas de Bernoulli, no Princípio dos Trabalhos Virtuais (PTV), desconsiderando-se nesses cálculos as forças por unidade de volume, implementados na plataforma do software Mathcad® versão 14. 
O elemento finito de barra possui dois graus de liberdade por nó, sendo uma translação e uma rotação, formulado com o uso de uma função polinomial interpolativa do terceiro grau.

A geometria aproximada para a peça é considerada linear e quadrática por partes. Na aproximação linear, empregadas na análise das madeiras Pinus caribaea, para cada duas circunferências sucessivas medidas, distantes L/8 umas das outras, utiliza-se um elemento finito, com momento de inércia definido pela eq.(2), sendo $h_{e} o$ comprimento do elemento finito, $x$ um número real variando no intervalo $\left[0, h_{e}\right]$ e $r_{i}$ e $r_{f}$ os valores dos raios à esquerda e à direita, respectivamente.

$$
I(x)=\frac{\pi}{4} \cdot\left(\frac{r_{f}-r_{i}}{h_{e}} \cdot x+r_{i}\right)^{4}
$$

O momento de inércia para a aproximação não linear da forma, empregado na análise das madeiras Corymbia citriodora, é expresso pela eq.(3), que relaciona a variação do raio, de forma quadrática, em função de seu comprimento, sendo $r_{i}, r_{m}$ e $r_{f}$, respectivamente, os valores dos raios iniciais, no ponto médio e no final de cada elemento finito.

$$
I(x)=\frac{\pi}{4 \cdot h^{16}} \cdot\left[2 \cdot\left(r_{f}+r_{i}-2 \cdot r_{m}\right) \cdot x^{2}+h \cdot\left(-r_{f}-3 \cdot r_{i}+4 \cdot r_{m}\right) \cdot x+h^{2} \cdot r_{i}\right]^{4}
$$

Os valores dos deslocamentos experimentais, assim como os valores dos diâmetros medidos ao longo do elemento (em função da forma de aproximação de sua geometria), são fornecidos aos programas EotmL e EotmN, com o objetivo de se calcular o efetivo valor do módulo de elasticidade da peça, intitulados $E_{o, L}$ e $E_{o, N}$, para as aproximações lineares e não lineares, respectivamente.

Cada programa, tendo por base os fundamentos do MEF, determina um vetor de deslocamentos numéricos $\left(U^{(n)}\right)$, contabilizando-se a influência das irregularidades existentes na geometria da peça, tendo como variável dependente o módulo de elasticidade do elemento estrutural.

Com posse do vetor de deslocamentos determinados pelos programas e do vetor de deslocamentos experimentais $\left(U^{(e)}\right)$, uma função é construída, fundamentada no Método dos Mínimos Quadrados (eq.(4)), cujo objetivo consiste na determinação do valor do módulo de elasticidade para que o resíduo gerado por ambas as soluções, numérica e experimental, seja mínimo, obtido pelo Método de Newton-Raphson.

$$
f(E)=\frac{1}{2} \sum_{i=1}^{n}\left(U_{i}^{(e)}-U_{i}^{(n)}\right)^{2}
$$

Como uma terceira forma numérica de calculo, depois de obtidos os módulos de elasticidade efetivos via programa EotmL para as madeiras Pinus caribaea, estes valores, juntamente com os referentes à geometria, condições de contorno, força e vinculações são utilizados para a simulação numérica das peças com elementos finitos tridimensionais, objetivando-se reproduzir os deslocamentos obtidos das experimentações. Estas simulações foram realizadas com o uso do software Abaqus versão 6.8.2, utilizando-se de um elemento finito tetraédrico de interpolação quadrática. Ressalta-se que as peças de madeira roliça quando não serradas (íntegras), como é o caso das madeiras aqui avaliadas, podem ser tratadas como material isotrópico (CHRISTOFORO et al., 2011). Pela falta de informações sobre o coeficiente de Poisson, optou-se por considerá-lo nulo nas simulações.

Com o objetivo de se verificar as diferenças existentes entre os valores dos módulos de elasticidade calculados, considerando-se o modelo simplificado $\left(\mathrm{E}_{\mathrm{deq}}\right)$ e as metodologias alternativas de cálculo propostas $\left(E_{o, L}\right.$ e $\left.E_{o, N}\right)$, foi utilizado o intervalo de confiança da diferença entre médias, expresso pela eq.(5), sendo; $\mu$ a média populacional das diferenças; $\bar{x}_{m}$ a média aritmética amostral das diferenças; $\mathrm{n}$ o tamanho da amostra; $\mathrm{S}_{\mathrm{m}}$ o desvio-padrão amostral das 
diferenças, e $t_{\alpha / 2, n-1}$ o valor tabelado da distribuição " $t$ " de Student, com n-1 graus de liberdade e nível de significância $\alpha$.

$$
\bar{x}_{m}-t_{\alpha / 2, n-1} \cdot S_{m} / \sqrt{n} \leq \mu \leq \bar{x}_{m}+t_{\alpha / 2, n-1} \cdot S_{m} / \sqrt{n}
$$

\section{RESULTADOS E DISCUSSÃO}

Os valores do $E_{d e q}$ e $E_{o, L}$ obtidos para as peças roliças estruturais de madeira Pinus caribaea são apresentados na Tabela 1, sendo DP o desvio-padrão e C.V. o coeficiente de variação.

TABELA 1. Valores do módulo de elasticidade obtidos para as madeiras Pinus caribaea. Values of the modulus of elasticity for Pinus caribaea round timber.

\begin{tabular}{cccccc}
\hline Peça & $\mathrm{E}_{\text {deq }}(\mathrm{MPa})$ & $\mathrm{E}_{\mathrm{o}, \mathrm{L}}(\mathrm{MPa})$ & Peça & $\mathrm{E}_{\mathrm{deq}}(\mathrm{Mpa})$ & $\mathrm{E}_{\mathrm{o}, \mathrm{L}}(\mathrm{MPa})$ \\
\hline 1 & 6.719 & 6.458 & 13 & 8.255 & 9.028 \\
2 & 8.455 & 8.516 & 14 & 6.935 & 7.311 \\
3 & 9.731 & 10.072 & 15 & 9.769 & 10.360 \\
4 & 8.466 & 8.127 & 16 & 9.078 & 8.738 \\
5 & 7.837 & 8.274 & 17 & 6.824 & 7.274 \\
6 & 7.288 & 6.836 & 18 & 5.976 & 6.228 \\
7 & 8.040 & 8.124 & 19 & 6.260 & 6.486 \\
8 & 7.720 & 8.260 & 20 & 9.829 & 10.382 \\
9 & 7.187 & 7.285 & 21 & 8.707 & 9.328 \\
10 & 6.900 & 7.211 & 22 & 7.557 & 6.916 \\
11 & 9.250 & 8.876 & 23 & 9.038 & 8.524 \\
12 & 8.872 & 8.459 & 24 & 6.597 & 6.437 \\
& & & Média & $7.970,42$ & $8.062,92$ \\
& & & DP & $1.159,70$ & $1.240,94$ \\
& & C.V. & 0,14 & 0,15 \\
\hline
\end{tabular}

O intervalo de confiança encontrado entre os valores do $E_{\text {deq }}$ e $E_{o, L}$ é $-790,79 \leq \mu \leq 605,79$ e, como o zero pertence ao subconjunto, constata-se que estes são estatisticamente equivalentes.

A regressão linear entre os valores do $E_{d e q}$ e $E_{o, L}(\mathrm{MPa})$ é ilustrada pela Figura 3, sendo a reta de ajuste e o coeficiente de correlação $\mathrm{R}^{2}$, respectivamente, iguais a $r(x)=1,00 \cdot x+50,48$ e 0,90.

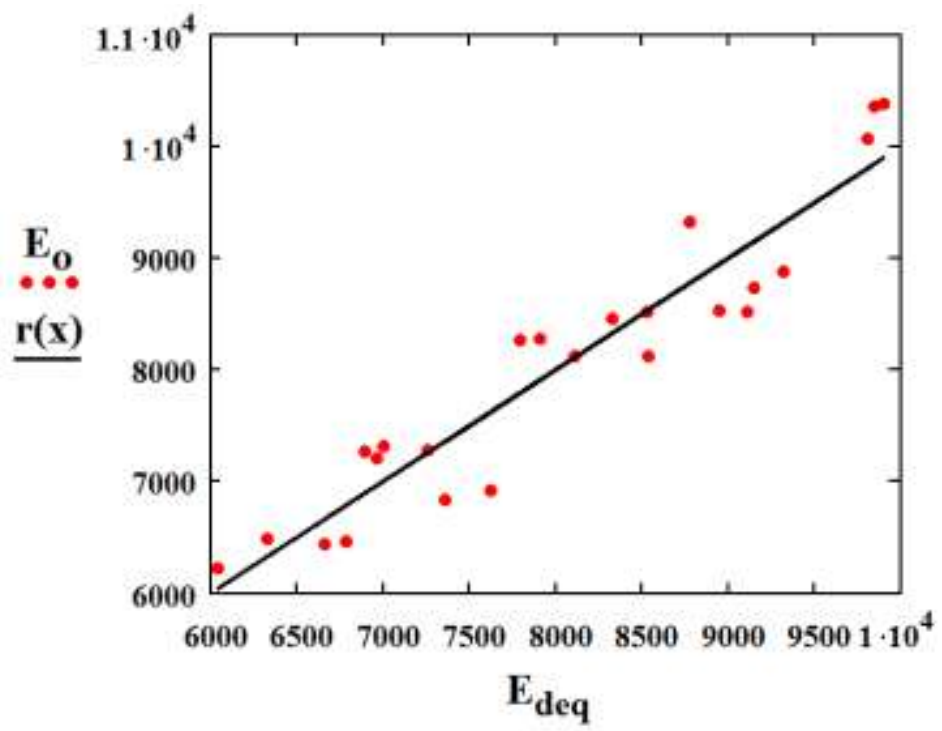

FIGURA 3. Correlação entre $E_{d e q}$ e $E_{o, L}$. Correlation between $\boldsymbol{E}_{d e q}$ and $\boldsymbol{E}_{\boldsymbol{o}, \boldsymbol{L}}$. 
Na Figura 4, ilustram-se os deslocamentos obtidos para a peça 1 (Tabela 1), segundo a modelagem com elementos finitos tridimensionais, com módulo de elasticidade efetivo $\mathrm{E}_{\mathrm{o}, \mathrm{L}}=6.458 \mathrm{MPa}$, força $4.520 \mathrm{~N}$ e comprimento 6,60 metros.

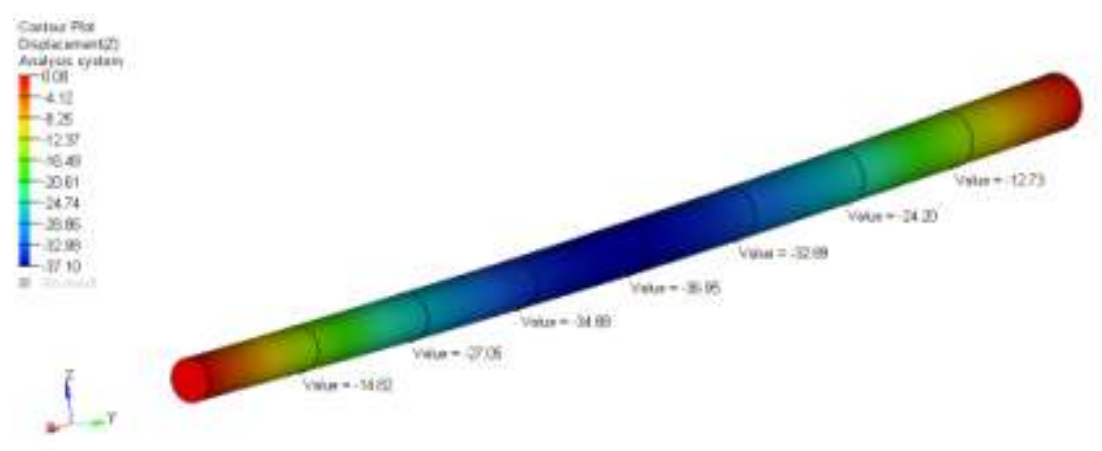

FIGURA 4. Deslocamentos obtidos para a peça 1. Displacements obtained for the element 1.

Na Tabela 2, apresentam-se os resultados em deslocamentos obtidos da experimentação com os deslocamentos obtidos pelo emprego de elementos finitos tridimensionais para a peça 1. Cabe chamar a atenção que os sinais negativos se justificam pela orientação de a força aplicada ter sentido contrário à do sistema de coordenadas utilizado no software.

TABELA 2. Deslocamentos experimentais e numéricos da peça 1. Experimental and numerical displacements of the structural element 1.

\begin{tabular}{ccccc}
\hline $\begin{array}{c}\text { Comprimento } \\
(\mathrm{mm})\end{array}$ & $\begin{array}{c}\text { Relógios } \\
\text { Comparadores }\end{array}$ & $\begin{array}{c}\text { Deslocamento Vertical - } \\
\text { Experimento }(\mathrm{mm})\end{array}$ & $\begin{array}{c}\text { Deslocamento Vertical - } \\
\text { Numérico 3D (mm) }\end{array}$ & Erro (\%) \\
\hline 0 & & 0 & 0 & \\
825 & $\mathrm{R} 1$ & $-12,20$ & $-12,73$ & $4 \%$ \\
1650 & $\mathrm{R} 2$ & $-23,08$ & $-24,20$ & $5 \%$ \\
2475 & $\mathrm{R} 3$ & $-31,11$ & $-32,89$ & $6 \%$ \\
3300 & $\mathrm{R} 4$ & $-33,30$ & $-36,95$ & $11 \%$ \\
4125 & R5 & $-33,07$ & $-34,88$ & $9 \%$ \\
4950 & R6 & $-26,00$ & $-27,05$ & $4 \%$ \\
5775 & R7 & $-14,94$ & $-14,82$ & $1 \%$ \\
6600 & & 0 & 0 & \\
\hline
\end{tabular}

Os resultados em deslocamentos da Tabela 2 são ilustrados na Figura 5.

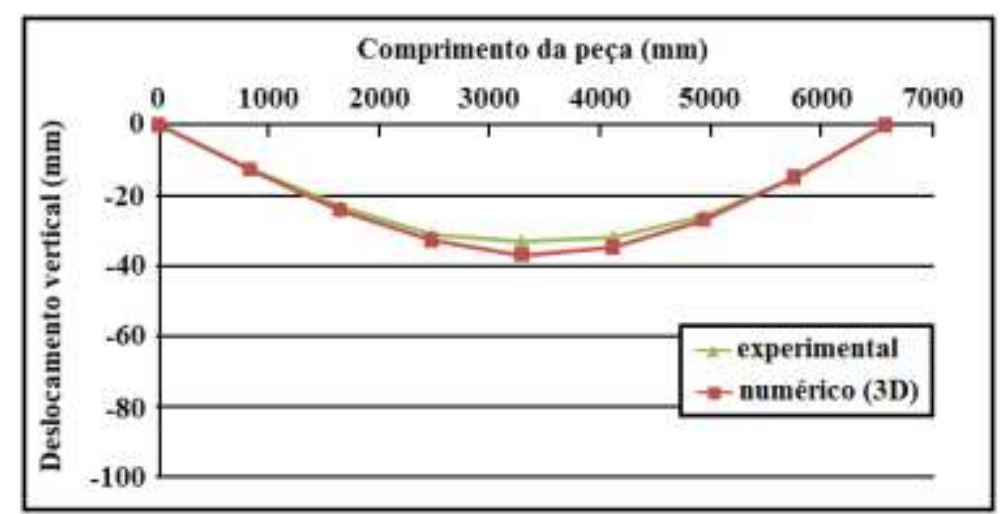

FIGURA 5. Comparação entre deslocamentos experimentais e numéricos (3D) para a peça 1. Comparison between experimental and numerical (3D) displacements for the structural element 1. 
Comparando-se os valores em deslocamentos experimentais com os valores de deslocamentos obtidos da simulação em 3D para as 24 peças roliças de madeira Pinus caribaea, os erros encontrados variaram de $0,85 \%$ a $12,33 \%$, sendo os maiores erros coincidentes na posição de maior deslocamento (ponto médio), indicando a boa aproximação do módulo fornecida pela simulação com elementos de barra de aproximação linear.

Os valores do $E_{d e q}$ e $E_{o, N}$ obtidos para as peças roliças estruturais de madeira Corymbia citriodora encontram-se na Tabela 3.

TABELA 3. Valores do módulo de elasticidade para a madeira Corymbia citriodora. Values of the longitudinal modulus of elasticity for Corymbia citriodora round timber.

\begin{tabular}{cccccc}
\hline Peças & $\mathrm{E}_{\text {deq }}(\mathrm{MPa})$ & $\mathrm{E}_{\mathrm{o}, \mathrm{N}}(\mathrm{MPa})$ & Peças & $\mathrm{E}_{\text {deq }}(\mathrm{MPa})$ & $\mathrm{E}_{\mathrm{o}, \mathrm{N}}(\mathrm{MPa})$ \\
\hline 1 & 20.432 & 21.423 & 13 & 21.928 & 22.663 \\
2 & 21.986 & 21.232 & 14 & 19.399 & 20.825 \\
3 & 19.799 & 21.174 & 15 & 19.251 & 18.946 \\
4 & 20.014 & 20.842 & 16 & 16.822 & 17.473 \\
5 & 17.858 & 19.332 & 17 & 16.209 & 15.791 \\
6 & 19.454 & 18.411 & 18 & 19.122 & 20.381 \\
7 & 18.321 & 19.614 & 19 & 19.345 & 21.016 \\
8 & 21.126 & 20.875 & 20 & 15.599 & 15.571 \\
9 & 21.207 & 20.238 & 21 & 16.595 & 18.023 \\
10 & 16.855 & 14.565 & 22 & 18.897 & 16.868 \\
11 & 15.506 & 16.237 & 23 & 16.512 & 14.475 \\
12 & 20.227 & 21.892 & 24 & 16.468 & 18.327 \\
& & & Média & $18.705,5$ & $19.008,1$ \\
& & & DP & $1.996,23$ & $2.414,25$ \\
& & & C.V. & 0,11 & 0,13 \\
\hline
\end{tabular}

O gráfico da regressão linear entre os valores dos módulos de elasticidade $E_{d e q}$ e $E_{o, N}(\mathrm{MPa})$ é ilustrado pela Figura 6, cuja equação de ajuste e o coeficiente de correlação $\left(\mathrm{R}^{2}\right)$ obtidos são, respectivamente, $r(x)=1,03 \cdot x-165,34$ e 0,72 .

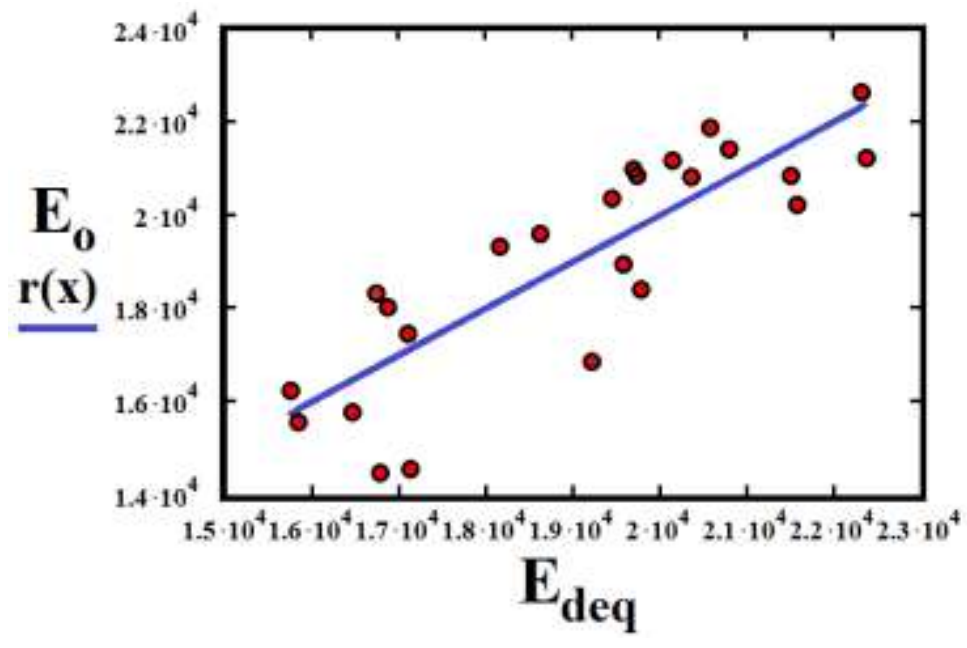

FIGURA 6. Regressão linear entre $E_{d e q}$ e $E_{o, N}$. Linear regression between $\boldsymbol{E}_{d e q}$ e $\boldsymbol{E}_{\boldsymbol{o}, \boldsymbol{N}}$.

$O$ intervalo de confiança entre os valores do $E_{\text {deq }}$ e $E_{0, N}$ é $-1.591,31 \leq \mu \leq 986,15$, sendo estatisticamente equivalentes pela pertinência do zero ao subconjunto encontrado. Isto implica que o modelo estrutural de ensaio de flexão estática a três pontos, juntamente com o uso da eq.(1) (modelo analítico), mostrou-se como boa alternativa de cálculo na determinação do módulo de 
elasticidade longitudinal também para as peças roliças estruturais de madeira Corymbia citriodora aqui estudadas. Ressalta-se que a equivalência encontrada entre os modelos de cálculo é valida apenas para as peças aqui estudas, de conicidade média 0,01 , podendo ser diferentes para outras peças e outras conicidades.

\section{CONCLUSÕES}

As boas aproximações obtidas entre os valores dos deslocamentos experimentais e numéricos (3D) indicam que o módulo de elasticidades efetivo $\left(E_{o L}\right)$, obtido pelo programa EotmL, com o uso de elementos finitos de viga, foi capaz de modelar a geometria das peças de forma satisfatória, sendo atraente do ponto de vista numérico, uma vez que a modelagem em três dimensões exige maior esforço computacional.

Pela análise estatística realizada sobre os valores do módulo de elasticidade longitudinal, para ambas as metodologias de cálculo utilizadas, analítica (eq.(1)) e numérica com elemento de barra, constata-se que as madeiras Pinus caribaea e Corymbia citriodora ensaiadas, em média, possuem geometria levemente cônica, podendo o módulo de elasticidade longitudinal ser obtido segundo o emprego direto da metodologia simplificada (equação analítica).

O emprego conjunto de ambas as formas de cálculo aqui apresentadas, analítica e numérica via elementos de barra, mostra-se como uma metodologia alternativa de classificação para o elemento roliço. Caso os resultados do módulo de elasticidade, para ambas as metodologias de cálculo, sejam estatisticamente equivalentes, isto implica que a peça apresenta pequena conicidade e geometria regular; caso contrário, a conicidade deve ser levada em consideração, e o valor do módulo de elasticidade da peça deve ser obtido com o emprego das estratégias numéricas de cálculo desenvolvidas.

\section{REFERÊNCIAS}

AMERICAN NATIONAL STANDARDS INSTITUTE. ANSI O5.1. American national standard for wood poles. Washington, 2002.

AMERICAN SOCIETY FOR TESTING AND MATERIALS. ASTM D1036. Standard test methods of static tests of wood poles. Philadelphia, 1997.

AMERICAN SOCIETY FOR TESTING AND MATERIALS. ASTM D198. Static tests of timbers in structural sizes. Philadelphia, 1976.

ASSOCIAÇÃO BRASILEIRA DE NORMAS TÉCNICAS. NBR 6122. Projeto e execução de fundações. Rio de Janeiro, 1996.

ASSOCIAÇÃO BRASILEIRA DE NORMAS TÉCNICAS. NBR 6231. Postes de madeira resistência à flexão. Rio de Janeiro, 1980.

ASSOCIAÇÃO BRASILEIRA DE NORMAS TÉCNICAS. NBR 7190. Projeto de estruturas de madeira. Rio de Janeiro, 1997.

ASSOCIAÇÃO BRASILEIRA DE NORMAS TÉCNICAS. NBR 8456. Postes de eucalipto preservado para redes de distribuição de energia elétrica. Rio de Janeiro, 1984.

ASSOCIAÇÃO BRASILEIRA DE NORMAS TÉCNICAS. NBR 8457. Postes de eucalipto preservado para redes de distribuição de energia elétrica - dimensões. Rio de Janeiro, 1984.

AUSTRALIAN STANDARD. AS 2209 - Timber - poles for over heads lines. Austrália. 1994.

BATISTA, A. M.; ROSSI, N.; MASCIA, N. T.; FURLANI, J. E. Estudo da flexão estática: relação entre peças de madeira com dimensões estruturais e dimensões reduzidas. In: ENCONTRO BRASILEIRO EM MADEIRAS E EM ESTRUTURAS DE MADEIRA, 7., São Carlos, 2000. 
CALIL JÚNIOR, C.; ESPINOSA, M. M.; MINÁ, A. J. S.; DIAS, A. A. Confiabilidade em elementos estruturais de madeira roliça. Revista Madeira, São Carlos, v.14, 2004. 1 CD-ROM.

CARREIRA, M. R.; DIAS, A. A. Avaliação da rigidez à flexão de toras de madeira por meio de vibração transversal. Cadernos de Engenharia de Estruturas, São Carlos, v.11, p.75-79, 2009. Online.

CHRISTOFORO, A. L.; PANZERA, T. H.; BATISTA, F. B.; BORGES, P. H.; ROCCO, F. A. L. Numerical evaluation of the longitudinal modulus of elasticity in structural round timber elements of Eucalyptus genus. Engenharia Agrícola, Jaboticabal, v.31, p.1.009-1.016, 2011.

COMITÉ EUROPÉEN DE NORMALISATION. DIN EN 14251. Structural round timbers - test methods. Bruxelas, 2004.

CORSINI, T. A.; FONTE, T. F.; CALIL JÚNIOR, C. Propriedades mecânicas de peças estruturais versus corpos de prova isentos de defeitos. In: ENCONTRO BRASILEIRO EM MADEIRAS E EM ESTRUTURAS DE MADEIRA, 9., 2004, Cuiabá.

LARSON, D.; MIRTH, R.; WOLFE, R. Evaluation of small-diameter ponderosa pine logs in bending. Forest Products Journal, Madison, v.54, p.52-58, 2004.

MINÁ, A. J. S. Estudo de estacas de madeira para fundações de pontes de madeira. 2005. Tese (Doutorado) - Escola de Engenharia de São Carlos, Universidade de São Paulo, São Carlos, 2005.

MINÁ, A. J. S.; DIAS, A. A. Estacas de madeira para fundações de pontes de madeira. Cadernos de Engenharia de Estruturas, São Carlos, v.10, p.129-155, 2008.

MINÁ, A. J. S.; DIAS, A. A.; CALIL JÚNIOR, C. Avaliação da rigidez e da resistência de postes de madeira para uso como estacas para fundações. In: ENCONTRO BRASILEIRO EM MADEIRAS E EM ESTRUTURAS DE MADEIRA, 9., 2004 Cuiabá.

PARTEL, P. M. P. Sistemas estruturais e construtivos utilizando madeira roliça de reflorestamento. 1999. Dissertação (Mestrado em Arquitetura e Urbanismo) - Escola de Engenharia de São Carlos, Universidade de São Paulo, São Carlos, 1999.

PINTO NETO, J.; CALIL JÚNIOR, C.; ESPINOZA, M. M. Propriedades de resistência e rigidez de peças estruturais roliças de pequeno diâmetro. In: ENCONTRO BRASILEIRO EM MADEIRAS E EM ESTRUTURAS DE MADEIRA, 9., Cuiabá, 2004.

RANTA-MAUNUS, A. Bending and compression properties of small diameter round timber. In: WORLD CONFERENCE ON TIMBER ENGINEERING, 2000, Whistler.

ROSS, R. J.; WANG, X.; MATTSON, J. A.; ERICKSON, J. R.; FORSMAN, J. W.; GESKE, E. A.; WEHR, M. A. Comparison of several nondestructive evaluation techniques for assessing stiffness and MOE of small-diameter logs. Madison. U. S. Department of Agriculture, Forest Service, Forest Products Laboratory, 2001. Research paper.

SALES, A.; CANDIAN, M. ; SALLES, V. C. Nondestructive evaluation of timber: the new Brazilian code for the design of timber structures. Materials and Structures, London, v.43, p.213221, 2010.

SALES, A.; PELIZAN, T. R.; OLIVEIRA, F. G. R.; CANDIAN, M.; LUCCHETTE, F. F.; SALGON, J. L.; MILLER, K. P. Avaliação de propriedades mecânicas de peças roliças de Eucalipto por meio de ultrassom. In: ENCONTRO BRASILEIRO EM MADEIRAS E EM ESTRUTURAS DE MADEIRA, 9., 2004, Cuiabá.

WOLFE, R.; MOSELEY, C. Small-diameter log evaluation for value-added structural applications. Forest Products Journal. Madison, v.50, p.48-58, 2000.

ZANGIÁCOMO, A. L.; ROCCO LAHR, F. A. Avaliação do efeito de cisalhamento na flexão de elementos roliços da espécie Eucalyptus citriodora. In: ENCONTRO BRASILEIRO EM MADEIRAS E EM ESTRUTURAS DE MADEIRA, 9., 2008, Londrina. 